\title{
Output and retrieval interference in the missing-number task
}

\author{
JEFFREY A. HADLEY and ALICE F. HEALY \\ University of Colorado, Boulder, Colorado \\ and \\ BENNET B. MURDOCK \\ University of Toronto, Toronto, Ontario, Canada
}

\begin{abstract}
Three experiments are reported that represent a reexamination of the missing-number method (Buschke, 1963b) of estimating short-term memory span. The missing-number task involved presenting a random sequence of all but one of the numbers of a known reference set and asking subjects to identify the missing number. Experiment 1 introduced a modified missing-number task that included two missing items and two choices made by the subject. With a large decline in performance for the second choice relative to the first, it is possible that only the second choice was subject to output or retrieval interference. An alternative explanation is that subjects output the number with the weakest memory representation as their first response. By postcuing subjects to report their two choices in a forward or backward sequence, Experiment 2 provided evidence against the importance of output interference and support either for the importance of retrieval interference or for the "weakest-first" hypothesis. However, with a paradigm that replaced only correctly identified missing numbers, a prediction that subjects would select the number with the weakest memory representation as their first response was not confirmed in Experiment 3. Instead, retrieval interference was implicated to explain the first-choice superiority found in Experiments 1 and 3. The results were interpreted in terms of the TODAM model of Murdock (1982, 1987, in press).
\end{abstract}

Researchers generally agree that the amount of information that one can retrieve in a memory span task is satisfactorily estimated by Miller's (1956) seven plus or minus two "chunks." For example, when a "chunk" consisted of words adjacent in both presentation and recall, Tulving and Patkau (1962) found that subjects were not able to utilize more than seven "adopted chunks" at a time. More recently, Ericsson (1985) found that without the use of long-term memory processes for encoding and retrieval, digit spans paralleled Miller's finding of about seven items. Despite a digit span that reached trained levels of 84 for 1 subject, when "meaningful encodings" (e.g., in terms of running times, dates, or years) were prevented by increases in presentation rate, or by the use of letters rather than numbers, his span dropped to the traditional size of about seven items.

This research was supported in part by United States Air Force Human Resources Laboratory Contract VE5744-022-001 and United States Army Research Institute Contracts MDA903-86-K-0155 and MDA90390-K-0066 to the Institute of Cognitive Science at the University of Colorado, Boulder, and by the Natural Sciences and Engineering Research Council of Canada Grant APA 146 to the University of Toronto. We wish to thank Steve Wallace for use of his computer laboratory in these experiments and Robert Crowder, Robert Greene, Margaret Intons-Peterson, Stuart Klapp, Michael Humphreys, David Mitchell, and an anonymous reviewer for their constructive comments on earlier versions of this manuscript. Requests for reprints should be sent to Alice F. Healy, Department of Psychology, University of Colorado, Campus Box 345, Boulder, CO 80309-0345.
The number of items that can be stored in immediate memory, though, remains a mystery. The effect of recalling an item on the recall of subsequent items, which Broadbent (1958) has labeled "output interference," is at the center of this dilemma. Not only can output interference be used to explain the superiority of items recalled first (see, e.g., Dalezman, 1976), it also serves to point out the inadequacies of methods that require retrieval of multiple items as a means of examining what is stored in memory. Despite the likelihood that one cannot recall as much as can be stored, the use of multiple-retrieval methods (i.e., methods that require the retrieval of multiple items) is widespread in testing individual differences. For example, Thorndike, Hagen, and Sattler (1986) include both forward and backward digit span tasks in a recent edition of the Stanford-Binet Intelligence Scale. Because the traditional memory span estimate includes the inhibiting effects of retrieval and output, it seems that a more valid measure of memory storage would be valuable from both a theoretical and an applied perspective.

The missing-number task developed by Buschke (1963a, 1963b; see Klapp, Marshburn, \& Lester, 1983, for a more recent use of this technique) was the result of one important attempt to overcome these problems of retrieval and output interference (other techniques that achieve the same purpose include, e.g., the probe method; see Klapp \& Netick, 1988). Buschke's technique involved randomly presenting all but one of the numbers of a known 
set and asking subjects to identify the missing number. With only one response per trial, retrieval and output interference were eliminated and a higher memory span estimate was proposed. In his first study, Buschke (1963b) compared the retrieval-free estimates from the missingnumber task with estimates from a digit span task in which subjects recalled, regardless of order, the presented numbers. He provided 15 trials in each of six different conditions that manipulated the range of numbers to be employed. He presented to all subjects $4,6,8,10,12$, or 14 numbers and contrasted the average number of trials correct, depending on whether a missing number was reported or whether all numbers had to be recalled. Utilizing a method described in Woodworth and Schlosberg (1954), Buschke (1963b) determined a stimulus threshold over subjects for each task, the means of which provided the estimates for the number of items retained. The resulting approximations yielded an average of 5.5 items retained for the digit span and 8.6 items retained for the missing-number task. This initial study confirmed the possibility that the missing-number task provides estimates of the storage characteristics of memory beyond those limited by the inhibiting effects of retrieval and output.

In a follow-up study, Buschke (1963a) applied the missing-number task to an investigation of serial position effects. By examining the errors of commission that occur when a previously presented item is reported as missing, one can generate a serial error curve. In this experiment, Buschke randomly presented 12 numbers from the range of 1 to 13 , one of which was missing. The probability of an error across serial positions ranged from near zero at the 12 th position to .15 at the $2 \mathrm{nd}$ and 4 th positions. Most important were the declining error probabilities that dropped from the 6th to the 12th serial positions.

Although the development of the missing-number task was an important accomplishment, this technique has been largely neglected in current memory research. The primary advantage of the missing-number task was, according to Buschke (1963a), the fact that it eliminated the harmful effects of output and retrieval. Because the inhibiting effect of recalling an item may be due either to the act of output per se or to processes of retrieval prior to output, we introduce a distinction in the present study between output interference (i.e., response effects) and retrieval interference (i.e., preresponse effects). Recently (see, e.g., Lewandowsky \& Murdock, 1989; Mewhort \& Popham, 1991; Reyna \& Brainerd, 1989), the concept of output interference has been used to account for a range of important memory phenomena. It has thus been shown that output and retrieval interference play crucial roles in the recall of presented items. But it is not known how (if at all) output and retrieval interference work in the identification of missing items. This question has been totally unexplored and at first sight might seem unanswerable. However, the missing-number task could be adapted for the study of output and retrieval interference effects by modifying the standard retrieval-free method developed by Buschke (1963a, 1963b). Specifically, one way to study output and retrieval interference would be to expand the missing-number technique to include more than a single missing number. For example, in the case of two missing numbers, the first response would be free of any effects of retrieval and output interference, and any drop in performance with the second response could be attributed to interference created by retrieving and outputting the first item. There are considerable data on the missing-one task, but there are no data at all that we know of on the missing-two task. Although the missing-two task may at first seem quite contrived, it is related to numerous everyday situations in which individuals try to determine which two (or more) items from a list are missing from a set. For example, a shopper often tries to determine which items from the grocery list are missing from the set included in the shopping cart, and a teacher often tries to determine which students from the class are missing from the set of those present at an examination. Exploration of the missing-two task should reveal interesting and important evidence about both the processes involved in identifying missing items and the interfering consequences of retrieval and output. The present experiments were conducted in order to provide such an exploration. In particular, the present study extended the missingnumber technique to the missing-two task in order to illuminate how the identification of missing items might come about and to examine whether output and retrieval interference play a role in the identification of missing items as they do in the recall of presented items.

\section{EXPERIMENT 1}

The first experiment was a simple extension of the missing-one task used by Buschke (1963a) to a case in which there were two missing items.

\section{Method}

Subjects. Thirty-six undergraduate students from an introductory psychology class at the University of Colorado participated in the experiment for course credit. They included 15 males and 21 females.

Procedures and Apparatus. Eleven numbers from 1 to 13 were sequentially presented on a VT 100 CRT screen at a 1 -sec rate, after which subjects orally reported their choices for the two missing numbers. In addition, after each response, a keyboand entry for computer storage was made by the experimenter. The presented numbers were arranged in random order so that (1) no consecutive numbers were in sequence, (2) each number from 1 to 13 occurred four times at each of the 11 serial positions, and (3) all numbers served as one of the missing numbers equally often. Each session began with the subject reading the following instructions:

\footnotetext{
This experiment will test your ability to detect which numbers are missing from a set of eleven numbers from 1 to 13 that will be individually shown on this screen in a random order. Your job will be to report verbally which two numbers were not presented. There is no time limit though please report your responses as soon as you are able. Once entered you cannot change your mind.
}

When the instructions were understood, two practice trials were performed to familiarize the subject with the task, whereupon the testing began. Fifty-two trials were given, with a 5-min break after 26 trials. The intertrial interval was 6 sec. A DEC PDP-11 com- 
Table 1

Percentage of Correct Responses for First and Second Choices in Experiments 1 and 3

\begin{tabular}{ccc}
\hline & \multicolumn{2}{c}{ Choice } \\
\cline { 2 - 3 } Experiment & First & Second \\
\hline 1 & 54.2 & 33.5 \\
3 & 54.4 & 35.9 \\
\hline
\end{tabular}

puter was used to control the experiment and provide an output of the raw data needed for analysis.

Design and analysis. Experiment 1 has a totally within-subject design with choice (first and second), presentation position (1-11), and actual number $(1-13)$ as the independent variables. Two sets of analyses were performed. First, the percentages of correct responses were examined across each choice. Second, presentation position and actual number were separately analyzed as a function of choice for error (of commission) frequency. Actual number was also analyzed with three additional dependent measures: (1) an overall frequency measure (without regard to whether correct or incorrect), (2) the percentage of correct responses for each number (given that it was used), and (3) an error (of omission) frequency.

\section{Results}

The percentage of correct responses for the first and second choices averaged over subjects is presented in Table 1 . A one-factor analysis of variance (ANOVA) with choice (first and second) as the independent variable was highly significant $\left[F(1,35)=137.5, M S_{\mathrm{e}}=56.1, p<\right.$ $.001]$. Each subject showed superior first-choice performance.

The choice $\times$ presentation position ANOVA for errors (of commission) produced main effects $[F(1,35)=140.2$, $M S_{\mathrm{e}}=1.3, p<.001 ; F(10,350)=29.7, M S_{\mathrm{e}}=2.9$, $\mathrm{p}<.001$, respectively $]$ and an interaction $[F(10,350)=$ $2.5, M S_{\mathrm{c}}=2.6, p=.007$ ] that were each highly significant. Figure 1 illustrates these results. Newman-Keuls post hoc tests revealed fewer first-choice errors (of commission) only at the 5th, 8th, 9th, 10th, and 11 th presentation positions.

The errors (of commission) for actual number showed a strong main effect $\left[F(12,420)=4.0, M S_{\mathrm{e}}=.3, p<\right.$ $.001]$, as well as a significant interaction with choice $\left.F(12,420)=4.7, M S_{\mathrm{e}}=.25, p<.001\right]$ (see Figure 2). The expected main effect of choice $\left[F(1,35)=148.3, M S_{c}\right.$ $=.1, p<.001]$ was significant. These effects of actual number can be best interpreted in terms of overall response frequency (regardless of accuracy). With a main effect of actual number $\left[F(12,420)=4.6, M S_{c}=2.3\right.$, $p<.001]$ and an interaction with choice $[F(12,420)=$ 7.6, $M S_{\mathrm{e}}=3.3, p<.001$ ] (see Figure 3), overall frequency showed a pattern of results similar to that found with commission errors. That is, on the first choice, lower numbers yielded more errors and greater overall frequencies, whereas on the second choice, higher numbers yielded more errors and greater overall frequencies. Consequently, given that a number was selected, the percentage of correct responses revealed no distinct advantages of any number. Figure 4 illustrates this result, in which the main effect of actual number was significant $[F(12,420)$ $\left.=2.7, M S_{e}=1,119.5, p=.002\right]$, but a Newman-Keuls post hoc test failed to show any significant paired comparisons. In addition, the expected main effect of choice was repeated $\left[F(1,35)=74.7, M S_{\mathrm{e}}=1,346.4, p<\right.$ $.001]$, and the choice $\times$ actual number interaction was not significant $[F(12,420)=1.1, p=.39]$. Finally, the distribution of omission errors across the ordinal sequence is shown in Figure 5. The main effect of actual number $\left[F(12,420)=3.7, M S_{e}=2.9, p<.001\right]$ was revealed in a Newman-Keuls post hoc test to be due primarily to the relative paucity of errors with the number 1 compared to all other numbers.

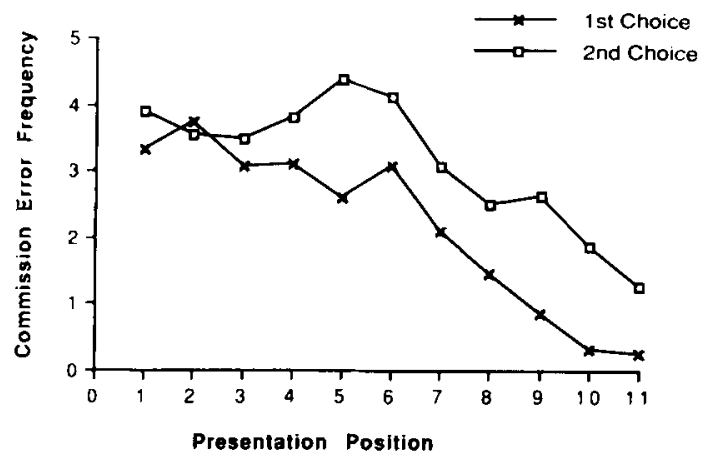

Figure 1. Error of commission frequency across presentation position for first choice and second choice in Experiment 1.



Figure 2. Error of commission frequency across actual number for first choice and second choice in Expertment 1.

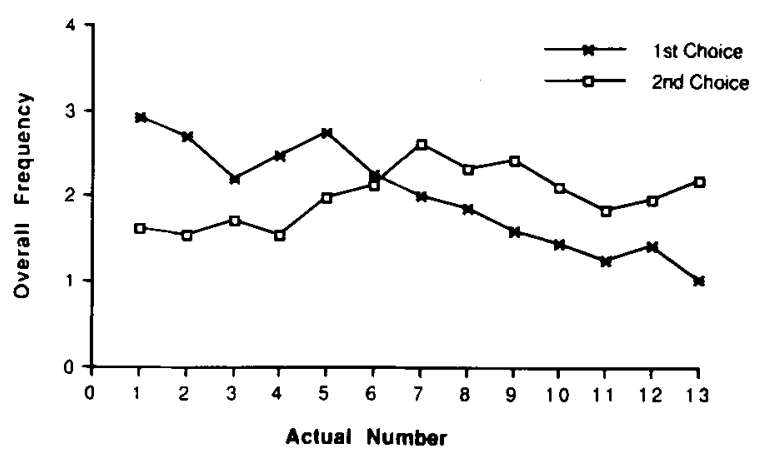

Figure 3. Overall frequency of actual number (correct and incorrect responses combined) for first choice and second choice in Experiment 1. 


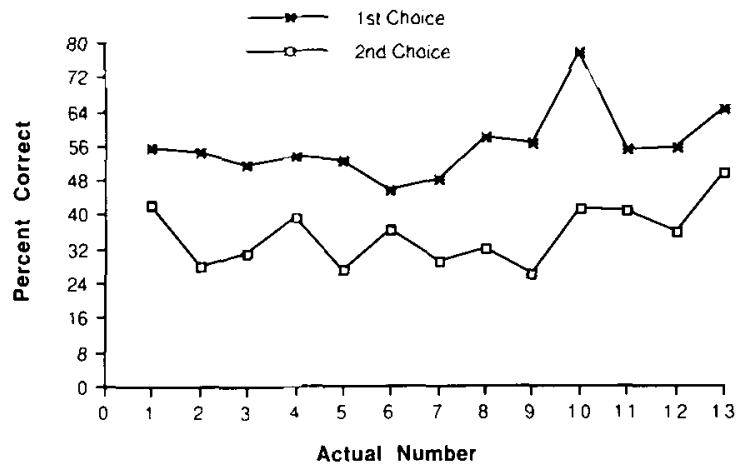

Figure 4. Percentage of correct responses for actual number, given that it was reported, for first choice and second choice in Experiment 1.

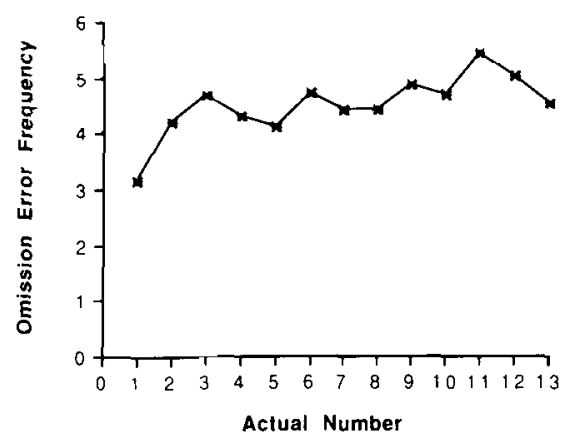

Figure 5. Error of omission frequency across actual number in Experiment 1.

\section{Discussion}

In Experiment 1, we were able to examine the combined effects of output and retrieval interference with the use of two missing items and two choices in the missingnumber task. If there are substantial effects of retrieval and output interference, the percentage of correct responses should be greater for the first than for the second choice. Only the response for the first choice should be free of the effects of output and retrieval interference. In contrast, the response for the second choice should be liable to interference related to outputting the response for the first choice. In fact, the percentage of correct responses was much higher for the first choice (54.2) than for the second choice (33.5), thereby documenting the large effects of output and retrieval interference. In addition, large differences in the distribution of commission errors across presentation position existed between first and second choices. First-choice errors were greatest at the early positions and dropped steadily to near zero at the two most recent positions. Second-choice errors also showed a recency effect; they were similar in frequency to first-choice errors at the early positions but greater in frequency at the middle and end positions. If the retrieval processes involved in responding with the first missing item did not interfere with the processes necessary to respond with the second missing item, then both choices should not only have resulted in equivalent percentages of correct responses but also have shown similar patterns of forgetting across presentation position. This outcome not being found, clear evidence for the problems associated with retrieval and output was demonstrated, and support was found for Buschke's (1963b) original proposal that the higher estimates of memory span found with the missing-number technique were due to an absence of retrieval and output interference.

Experiment 1 also provided some other support for these conclusions with an analysis of actual number. Because of past findings that fewer errors occurred nearer the ends than the middle of the ordinal sequence (Buschke \& Hinricks, 1968; Buschke \& Lenon, 1969), researchers concluded that presented items must have been perceived and encoded in terms of an ordinal sequence (see also Madsen \& Drucker, 1966). Experiment 1, though, could not support these conclusions. Reliable changes in error frequency did occur across actual number (and differently for first and second choices). But very similar changes also occurred in the overall frequency of number use (when correct responses were combined with incorrect responses). According to this analysis, subjects tended to report as the first choice a number from the beginning of the ordinal sequence and as the second choice a number from the end of the ordinal sequence. However, when reported, no numbers had a reliable advantage to being correct. In a similar result, an analysis of omission errors also failed to agree with past research that showed increasing errors toward the middle of the ordinal sequence in this task. Because the numbers reported as well as the numbers missed had little influence on the probability of a correct or an incorrect response, respectively, it seems doubtful that an encoding strategy that focused on ordinal sequence was responsible for the effects of actual number. Rather, these effects of actual number, which differed dramatically for the first and second choices, seem attributable entirely to retrieval and output factors. For example, the retrieval process may include a search of the ordinal sequence from low to high.

In summary, a lack of retrieval and output interference may have been the primary cause of the large and consistent performance differences found from first to second choice. In addition, an analysis of actual number also provided evidence that an encoding strategy based on ordinal sequence may not play a dominant role in determining first-choice performance. Instead, the drop in performance with the second choice strongly suggested that superior first-choice performance reflected the number of items one can report when free from retrieval and output interference, reestablishing Buschke's (1963a, 1963b) missing-number procedure as an optimal method of estimating the span of immediate memory.

\section{EXPERIMENT 2}

Experiment 1 examined the effects of retrieval and output interference in the missing-number task. When asked to select two missing numbers, subjects in Experiment 1 revealed inferior performance on the second choice. How- 
ever, an alternative explanation for this pattern of results is possible. Rather than resulting from retrieval and output interference, the performance change from first to second choice may have been the result of subjects' simply using the best choice as the first response. In an ordinary freerecall task, the best choice would be the one with the strongest memory representation (but see Brainerd, Reyna, Howe, \& Kevershan, 1990). However, in the missingnumber task, in which subjects respond with the numbers not presented on a trial, the best choice would be the one with the weakest memory representation. This "weakestfirst" hypothesis could account for the first-choice superiority found in Experiment 1. Experiment 2 was designed to check for this possibility. Subjects were postcued to output (but not necessarily to retrieve) their two choices in either a forward or a backward sequence, which forced them to divide the best responses (i.e., the items with the weakest representations) between first and second choices. Any remaining first-choice superiority that might occur with both forward and backward report orders could only be due to difficulties associated with output per se. On the other hand, a disappearance of the performance differences between first and second choices would support the weakest-first hypothesis and would rule out an explanation in terms of output interference. (This finding would also be inconsistent with any explanation involving a loss of information as a function of time.) At the same time, such a result would be consistent with an explanation in terms of retrieval interference. Given that the retrieval process itself is insensitive to ordinal sequence, the necessity to output the missing numbers in a prescribed order would result in the first item retrieved reported equally often in the first and second positions. Thus, a disappearance of the first-choice superiority in both forward and backward report orders would also be predicted by the retrieval interference explanation. Therefore, this experiment might allow for the separation of the effects of output interference from the effects of retrieval interference.

Recall that in Experiment 1 the order of the two choices selected by subjects did not appear to be random; rather, the first choice tended to be a lower number than the second choice. Contrary to the suggestion above, this finding can be interpreted as consistent with a retrieval process that is sensitive to ordinal sequence (i.e., with a search of the ordinal sequence from low to high). Given this possibility, the retrieval interference explanation would now predict a first-choice superiority with the forward report order (in which retrieval order parallels output order) but a second-choice superiority with the backward report order (in which retrieval order is opposite to output order). Furthermore, because the item with the weakest memory representation would presumably be independent of the ordinal sequence (i.e., all numbers from 1 to 13 occurred equally often as missing numbers), such a choice $\times$ report order interaction would also rule out the weakest-first hypothesis. In contrast, if no difference is found between the two report orders, evidence would be provided that the subsequent output process, rather than the retrieval process, was responsible for the subjects' tendency in Experiment 1 to report their two choices from low to high. In other words, subjects may retrieve the two missing numbers in a manner that is independent of their relative positions in the ordinal sequence, but after the two numbers have been retrieved, they may output them in numerical order.

\section{Method}

Subjects. Thirty-six undergraduate students from an introductory psychology class at the University of Colorado participated in Experiment 2 for course credit. They included 17 males and 19 females.

Procedure and Apparatus. Except for the postcue for the required report order, all procedures and apparatus were identical to those employed in Experiment 1. One second after the last number in the presentation, the words "FORWARD" or "BACKWARD" were flashed briefly to cue the subject.

Design and analysis. Experiment 2 had a totally within-subject design with choice (first and second), presentation position (1-11), actual number (1-13), and order of report (forward and backward) as the independent variables. Three sets of analyses were performed. First, a choice $X$ order of report ANOVA was conducted, with percentage of correct responses as the dependent variable. Second, a choice $x$ presentation position $x$ order of report ANOVA was performed, with error (of commission) frequency as the dependent measure. Last, actual number was analyzed with two dependent measures that were found in Experiment 1 to provide the best means of comparing performance across ordinal positions. First, success in reporting a particular number was determined, unconfounded by how many times that number had been attempted, and a choice $x$ actual number ANOVA was conducted with the percentage of correct responses (given that they were reported) as the dependent measure. In addition, examination of how many occasions each number was overlooked (when missing) resulted in an error (of omission) frequency that provided a second analysis of actual number.

\section{Results}

The percentage of correct responses at each report order for the first and second choices averaged over subjects is presented in Table 2. The two-factor ANOVA on choice (first and second) and order of report (forward and backward) revealed no main effects or interactions. All $F$ ratios were less than 1 .

The choice $\times$ presentation position $\times$ order of report ANOVA for errors (of commission) produced a main effect of presentation position $\left[F(10,350)=20.2, M S_{\mathrm{e}}=\right.$ $1.3, p<.001]$. Figure 6 illustrates this effect and the significant interaction between choice and presentation position $\left[F(10,350)=2.5, M S_{e}=1.4, p=.007\right]$. Although a Newman-Keuls post hoc test allowed an interpretation of the main effect of presentation position as a steady drop in the errors (of commission) from the 6th to 11th posi-

Table 2

Percentage of Correct Responses for First and Second Choices at Eech Report Order in Experiment 2

\begin{tabular}{lcc}
\hline \multirow{2}{*}{ Report } & \multicolumn{2}{c}{ Choice } \\
\cline { 2 - 3 } Order & First & Second \\
\hline Forward & 41.9 & 41.6 \\
Backward & 42.5 & 39.6 \\
\hline
\end{tabular}




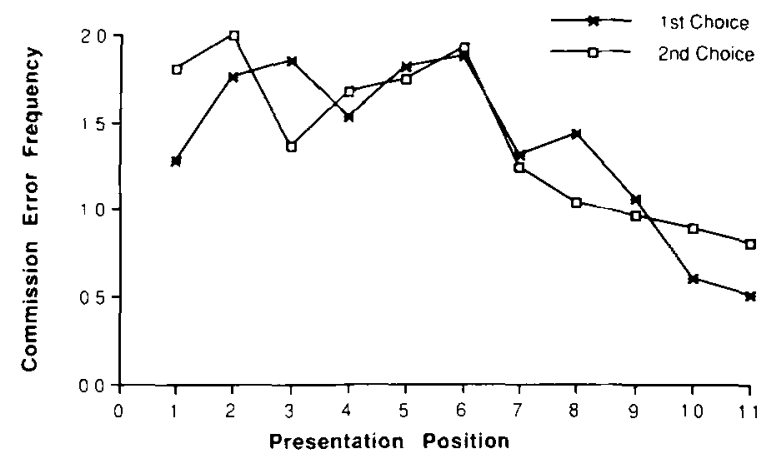

Figure 6. Error of commission frequency across presentation position for first choice and second choice in Experiment 2.

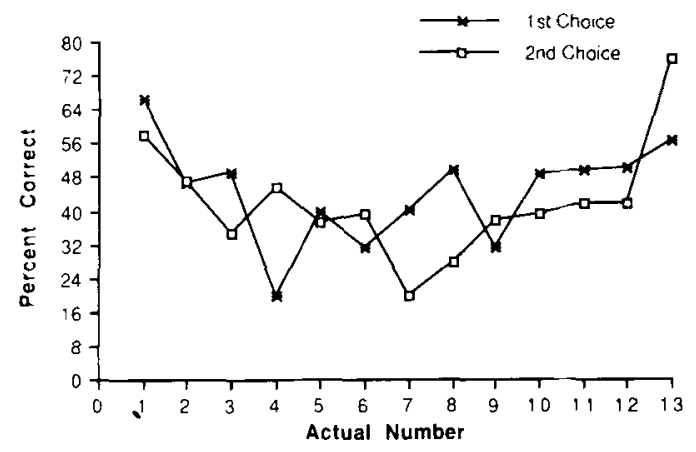

Figure 7. Percentoge of correct responses for actual number, given that it was reported, for first choice and second choice in Experiment 2.

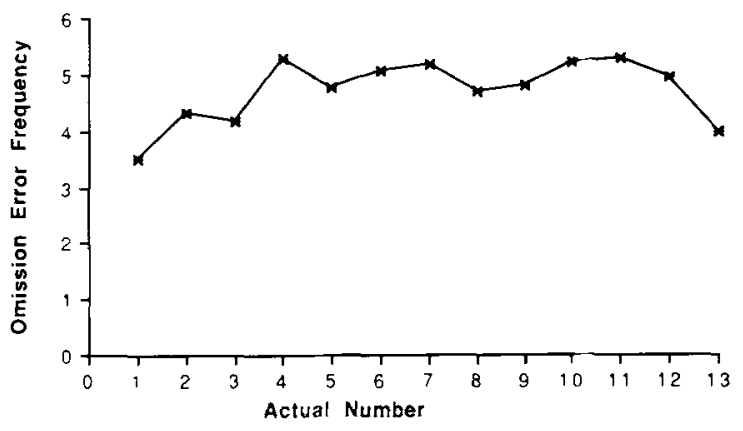

Figure 8. Error of omission frequency across actual number in Experiment 2.

tions, the significant interaction of choice and presentation position was more difficult to interpret. That is, although the interaction was apparently due to a combination of: (1) fewer first-choice errors on the 1st, 10th, and 11th positions, and (2) fewer second-choice errors on the 3rd and 8th positions, none of the paired comparisons across choice at any of the presentation positions reached a .05 level of significance in Newman-Keuls post hoc tests. The main effects for choice and order of report, as well as all interactions with order of report, were nonsignificant.
The analysis of actual number for percentages of correct responses (given that they were reported) revealed a main effect $\left[F(12,420)=12.3, M S_{e}=679.9, p<\right.$ $.001]$ and an interaction with choice $[F(12,420)=6.2$, $\left.M S_{\mathrm{e}}=600.7, p<.001\right]$ (see Figure 7). The NewmanKeuls post hoc tests showed that: (1) the main effect of number occurred as a result of superior performance with the numbers 1 and 13, and (2) the choice $\times$ actual number interaction was due to the fact that the numbers 7 and 8 had superior first-choice performance, whereas the numbers 4 and 13 had superior second-choice performance. The distribution of omission errors across ordinal position is shown in Figure 8. In this case, the main effect of actual number $\left[F(12,420)=4.4, M S_{\mathrm{e}}=2.5, p<\right.$ $.001]$ was attributed, by means of a Newman-Keuls post hoc test, to the fact that the number 1 had fewer errors than each of the numbers $4,6,7,10,11$, and 12 .

\section{Discussion}

It is clear that the practice of postcuing subjects to report their two choices in either a forward or a backward sequence was an effective means of distinguishing between an output interference explanation for the superior firstchoice performance in Experiment 1, on the one hand, and either a retrieval interference or a weakest-first explanation, on the other. That is, without causing any main effects or interactions, this manipulation had a strong effect on the relative performances of first and second choices. For both forward and backward report orders, the percentage of correct responses for the first choice was equivalent to that for the second choice. In addition, although the error (of commission) frequency across presentation position was very different with each choice in Experiment 1, in Experiment 2 these differences between first- and second-choice errors were greatly diminished.

Apparently, when subjects were forced to divide their best answers (as determined by either the weakest-first or the retrieval interference account) between first and second choices, the difference between the two choices was minimized. Hence, a performance tradeoff occurred, and evidence against the output interference explanation for the first-choice superiority in Experiment 1 was found. The present results suggest that the first-choice superiority found in Experiment 1 was due instead either to retrieval interference or to a weakest-first strategy (in which subjects order their output according to some inverse function of memorial strength).

Although the subjects in Experiment 1 tended to report the two missing numbers according to their ordinal sequence, the forward and backward report orders did not yield performance differences in Experiment 2. This finding is inconsistent with the suggestion from Experiment 1 that the retrieval process includes a search of the ordinal sequence from low to high. The tendency to report the lower number first in Experiment 1 must, therefore, have been a consequence of output, rather than retrieval, factors. That is, subjects may have retrieved the items in an order that was independent of their positions in the ordinal sequence, but they chose to output them from low to high. 
Before any definitive conclusions can be made about the weakest-first strategy, the possibility of retrieval interference must be considered. Although the paradigm used in Experiment 2 controlled for output order, it could not control for retrieval order and therefore failed to eliminate a retrieval interference explanation for the firstchoice superiority found in Experiment 1. For example, if the number retrieved first was too high or too low for a forward or backward report order, respectively, it would likely have been withheld by the subject until a more appropriate (yet perhaps inferior) first choice could be retrieved. Therefore, if a particular choice is better because it is retrieved first (as opposed to being retrieved first because it is better), this task-related phenomenon in which output order would not equal retrieval order may have been the cause of the lack of difference between first and second choices in Experiment 2. In other words, although evidence was presented against the hypothesis that a first response could be better simply because it was output first, it remained possible that a retrieval-based interference phenomenon might provide an explanation for the first-choice superiority found in Experiment 1. Unfortunately, though, due to an inability to observe the process of retrieval per se directly, it seemed necessary to test the weakest-first hypothesis further. By returning to a constraint-free report order (in which output order would be likely to coincide with retrieval order), in Experiment 3 we attempted to provide a more refined explanation for the first-choice superiority found in Experiment 1.

\section{EXPERIMENT 3}

Experiment 3 tested the weakest-first hypothesis. According to that hypothesis, when report order is unconstrained, subjects select as their first response the item with the weakest memory representation. In a systematic replication of Experiment 1, the numbers presented on each trial were varied so that a manipulation of memory strength or familiarity was possible. Only the missing items that were correctly identified were replaced, so that memory strength could be judged by the number of consecutive trials on which an item had remained missing. In other words, when a number is missing on the preceding trial, its memory representation should be weaker than when it is presented on the preceding trial. Hence, contrary to the situation with the standard recall task, repetition of a missing number should decrease, rather than increase, its memory strength. Support for a weakest-first hypothesis requires three separate findings. First, a reestablishment of the performance differences between first and second choices is necessary. Second, repetition of missing numbers must be shown to benefit performance on the missing-number task. And third, any beneficial effects of repetition must be weighted in favor of first-choice performance.

\section{Method}

Subjects. Thirty-six undergraduate students from an introductory psychology class at the University of Colorado participated in Experiment 3 for course credit. They included 18 males and 18 females.

Procedure and Apparatus. As in Experiments 1 and 2, 11 numbers from 1 to 13 were individually presented on a VT100 CRT screen at a $1-\mathrm{sec}$ rate, after which subjects orally reported their best choices for the two missing numbers. Although all task variables and instructions to the subject were identical to those used in Experiment 1 , the procedures for determining the missing numbers in each trial were unique to the present experiment. A process was carried out in which unreported missing numbers remained missing in subsequent trials; replacement of any missing number was not made until it was reported by the subject. For example, if 3 and 10 were missing and 8 and 10 were reported, 3 and a randomly selected number (other than 10) would be missing on the next trial. In addition, although all subjects performed at least 52 trials, some subjects required additional trials to ensure that each number from 1 to 13 was used as a replacement three times. As a result, although the total number of trials performed ranged from 52 to $63(M=$ $53.1, S D=3.0$ ), the number of trials that made possible a meaningful analysis of actual number ranged from 28 to $63(M=43.8$, $S D=8.9$ ). All other dependent measures were based on the 52 trial score used in previous experiments.

Design and analysis. Experiment 3 had a totally within-subject design with choice (first and second), presentation position (1-11), actual number (1-13), and missing age (1-17) as independent variables. With the exception of the changes instituted in Experiment 2 for the analysis of actual number, all statistical arlalyses used in Experiment 1 were performed in Experiment 3. In addition, with missing-number "age" defined by how many consecutive trials an item had been missing (missing numbers began with an age of 1 and got "older" with each trial on which they were not chosen), several other analyses described in the results section were included to determine the effect of age on missing-number performance. Last, three analyses were conducted to examine whether the older (and weakest) item was more likely to be selected on the first choice. By assigning an age of zero to presented items (i.e., commission errors), a one-factor ANOVA with choice as the independent variable and response age as the dependent variable was possible. Second, a one-factor ANOVA was completed with response age also as the dependent measure, but only on trials on which both choices were correct. And third, only trials with one correct response were considered, so that an analysis was possible that compared the age of the chosen number with the age of the overlooked number in a two-factor ANOVA that crossed choice (when correct on the first choice and when correct on the second choice) with missing number (reported and nonreported).

\section{Results}

The percentage of correct responses for the first and second choices averaged over subjects is presented in Table 1. A one-factor ANOVA on choice yielded a highly significant effect, with superior first-choice performance on percentage of correct responses $\left[F(1,35)=103.6, M S_{\mathrm{e}}\right.$ $=59.3, p<.001]$.

The choice $\times$ presentation position ANOVA for errors (of commission) produced main effects $[F(1,35)=107.2$, $M S_{\mathrm{e}}=1.4, p<.001$, and $F(10,350)=26.8, M S_{\mathrm{e}}=$ $2.6, p<.001$, respectively $]$ and an interaction $[F(10,350)$ $\left.=3.1, M S_{\mathrm{e}}=2.7, p=.001\right]$ that were each highly significant. Figure 9 illustrates these effects. A NewmanKeuls post hoc test showed: (1) a main effect of presentation position, in which errors remained high and equivalent from the 1st through 5th positions before dropping steadily to near zero at the most recent presentation position, and (2) a choice $\times$ presentation position interaction, 


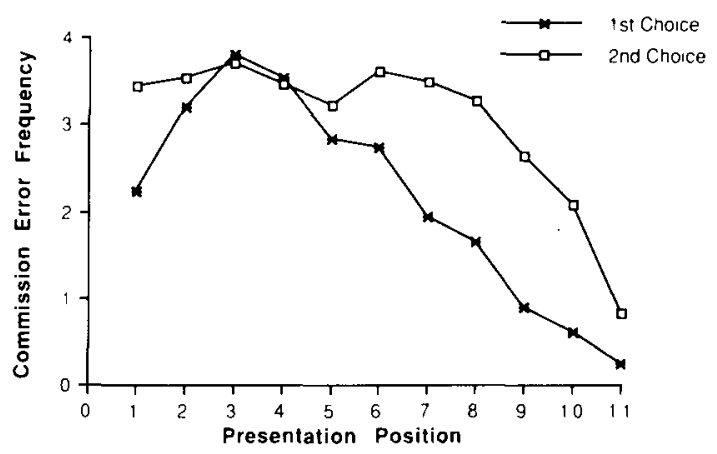

Figure 9. Error of commission frequency across presentation position for first choice and second choice in Experiment 3.

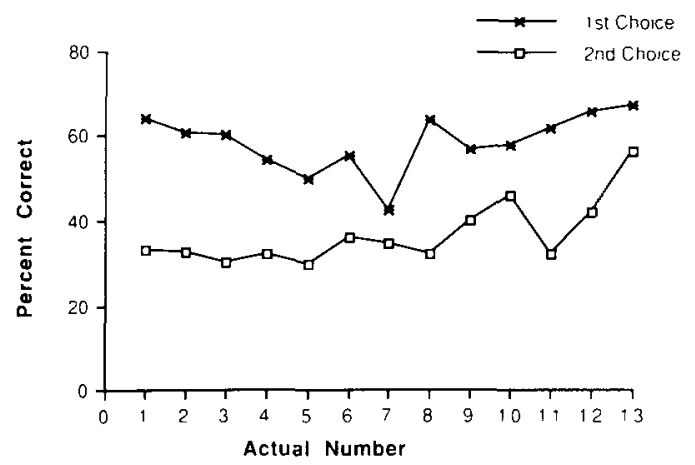

Figure 10. Percentage of correct responses for actual number, given that it was reported, for first choice and second choice in Experiment 3.

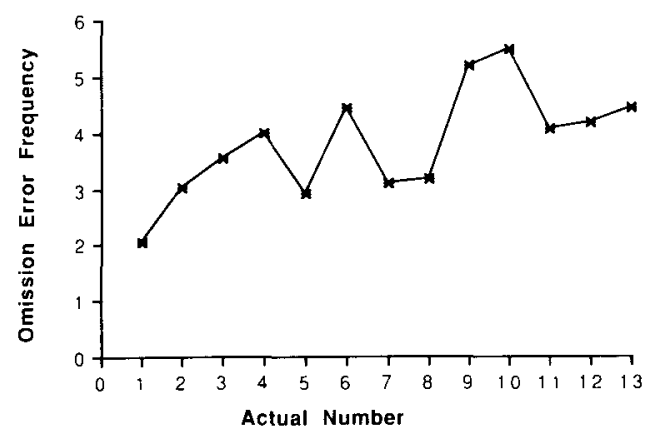

Figure 11. Error of omission frequency across actual number in Experiment 3.

which was due to fewer first-choice errors at only the 7th through 10th positions.

The analysis of actual number for percentage of correct responses revealed few distinct advantages of any number (given that it was chosen). Figure 10 illustrates this result, in which a main effect of actual number $\left[F(12,420)=4.2, M S_{\mathrm{e}}=622.9, p<.001\right]$ was interpreted by a Newman-Keuls post hoc test as a superiority of the final number 13 over the other numbers. The choice $X$ actual number interaction was not significant $[F(12,420)$ $=1.3, p=.20 \mathrm{~J}$, although the main effect of choice was significant $\left[F(1,35)=98.1, M S_{\mathrm{e}}=1,139.1, p<.001\right]$.
The distribution of omission errors across the ordinal sequence is shown in Figure 11. The main effect of actual number $\left[F(12,420)=2.7, M S_{\mathrm{e}}=12.4, p=.002\right]$ was shown by a Newman-Keuls post hoc test to be due to a lack of errors with the number 1 in comparison with all other numbers.

As a result of the replacement procedures, the mean age difference between the two missing alternatives on each trial was 1.9 and the standard deviation was 1.0 . Out of a total of 3,744 missing numbers used, 1,769 numbers were introduced with an age of one, 1,688 missing numbers were eventually reported, and 81 were left unreported at the completion of the experimental session.

Patterned after an analysis used by Murdock and Babick (1961), an expected frequency distribution across age, which would occur if repetition had no effect on performance, was compared with an observed frequency distribution in a chi-square goodness-of-fit test. That is, given that performance was independent of all previous trials, the probability of reporting a missing item (with $p$ estimated from the observed mean age of a missing number) would equal $p$ at the age of one, $p(1-p)$ at the age of two, $p(1-p)^{2}$ at the age of three, and thus, more generally (with $n$ as missing age), the geometric distribution described by $p(1-p)^{n-1}$. These expected probabilities were multiplied by the total number of missing items reported by all subjects, and the resulting geometric distribution was tested against the obtained distribution. More specifically, on the basis of the mean age of a missing number, $p$ was estimated at .483 , and this proportion was used to predict how the 1,688 correct responses would be distributed across missing age (if age had no effect on performance). For example, the expected frequency of correct responses at the age of three would equal the total number of correct responses $(1,688)$ multiplied by its expected probability of a correct response $\left[.483(1-.483)^{2}=\right.$ $.129]$ and thus would equal approximately 218 correct responses.

The obtained and expected frequency distributions across missing age are presented in Table 3. Although the resulting chi-square goodness-of-fit was significant $\left[\chi^{2}(8, N=36)=16.25, p<.05\right]$, the differences between obtained and expected frequency distributions did not illustrate a beneficial effect of repetition. Contrary to

Table 3

Observed and Expected Frequency Distributions of Correct Responses Across Missing Age

\begin{tabular}{ccc}
\hline Age & Observed & Expected \\
\hline 1 & 847 & 815.3 \\
2 & 432 & 421.5 \\
3 & 181 & 217.9 \\
4 & 99 & 112.7 \\
5 & 59 & 58.2 \\
6 & 25 & 30.1 \\
7 & 19 & 15.6 \\
8 & 12 & 8.0 \\
$9-17$ & 14 & 8.7 \\
& 1,688 & 1,688 \\
\hline
\end{tabular}


a case in which correct responses occur earlier than expected, the significant chi-square was due to several numbers that remained missing longer than expected. However, because this chi-square did not employ independent observations (there were entries from each subject at each age category), the differences between expected and obtained frequencies were also examined across subjects. A (obtained minus expected) signed difference measure for each subject was calculated at each age category, and a series of independent $t$ tests were conducted. Table 4 presents the $t$-test results at each missing age in which only the age categories of 9,11, and 16-17 displayed obtained minus expected mean differences that were significantly different from zero. Whereas the significant chisquare seemed to suggest a serious discrepancy, an examination of these $t$ tests revealed that the majority of correct responses were distributed across missing age in a very similar manner to what would be expected if repetition had no effect on performance.

An additional analysis that compared obtained and expected frequency distributions was also taken from Murdock and Babick's (1961) study. Because the average number of trials necessary to make a correct response of a missing number-assuming a geometric distributionis equal to the reciprocal of the overall probability of a correct response (e.g., with a probability of a correct response of $25 \%$, subjects would be expected to have an average of one correct response every four attempts or a mean correct age equal to $1 / .25$ ), a signed difference measure between obtained and expected mean correct ages was also examined in a $t$ test. A significant $t$ test that displayed a mean difference measure significantly less than zero would support a beneficial effect of age on missingnumber performance. Instead, however, although a tendency did exist in this direction (obtained and expected mean correct ages were 2.2 and 2.3 , respectively), the $t$ test was not significant $[t(35)=-1.1, p=.17]$.

The examination of missing age across choice yielded one significant finding. Although response age did result

Table 4

Observed Minus Expected Mean Differences in Correct Frequency With Standard Errors and $t$-Test Results Presented at Each Missing Age

\begin{tabular}{cccc}
\hline Age & $M$ & $S E$ & $t(35)$ \\
\hline 1 & 0 & & \\
2 & .9 & 1.81 & .48 \\
3 & -.6 & .61 & -.95 \\
4 & -.2 & .39 & -.51 \\
5 & .04 & .29 & .15 \\
6 & -.2 & .15 & -1.25 \\
7 & .03 & .11 & .24 \\
8 & .07 & .04 & 1.78 \\
9 & -.1 & .007 & $20.58 \dagger$ \\
10 & .01 & .02 & .56 \\
11 & .02 & .01 & $2.04^{*}$ \\
$12-13$ & .02 & .01 & 1.65 \\
$14-15$ & .005 & 4.01 & 1.32 \\
$16-17$ & .05 & .009 & $5.10 \dagger$ \\
\hline${ }^{*} p<.05$. & $\dagger p<.001$. & &
\end{tabular}

Table 5

Mean Age of Reported and Nonreported Missing Numbers When Correct Oniy on Either First or Second Choice in Experiment 3

\begin{tabular}{lcc}
\hline Correct & \multicolumn{2}{c}{ Missing Number } \\
\cline { 2 - 3 } Choice & Reported & Nonreported \\
\hline First & 1.10 & 1.38 \\
Second & 1.25 & 1.27 \\
\hline
\end{tabular}

in a main effect of choice $\left[F(1,35)=30.8, M S_{\mathrm{e}}=0.1\right.$, $p<.001]$ in which first choice was older than second choice (first and second choice mean response ages were 1.1 and .8 , respectively), this outcome was probably a simple consequence of the fact that there were more correct responses on the first choice, and it was not necessarily related to a tendency by subjects to select the oldest item on the first response. As a result, when response age was examined only for trials on which both choices were correct, no main effect of choice was found $[F(1,35)<1]$, and the first choice tended to be younger than the second choice (first and second choice mean response ages were 1.0 and 1.2 , respectively). Similarly, on trials with only one correct response, the examination of missing age in a choice (when correct on the first choice and when correct on the second choice) $\times$ missing number (reported and nonreported) ANOVA revealed no main effects $[F(1,35)$ $<1$ and $F(1,35)=2.8, p=.10$, respectively] and no interaction $[F(1,35)=1.4, p=.24]$. As shown in Table 5 , two important trends did emerge. First, the nonreported missing number tended to be older than the reported missing number. And second, this finding tended to occur primarily when subjects were correct on the first choice rather than the second choice.

\section{Discussion}

The primary result from Experiment 3 is that the replacement procedure in which subjects were given as many trials as necessary to report each missing number failed to benefit performance on the missing-number task. In fact, the comparison of the obtained frequency distribution across missing age with the expected frequency distribution (assuming that repetitions did not affect performance) resulted in a significant chi-square goodness-of-fit in which several missing numbers were reported later than expected. Hence, the repetition of missing numbers actually appeared detrimental to performance on the missingnumber task. At the same time, though, additional analyses indicated that the majority of correct responses were distributed across missing age similarly to what would be expected if repetition had no effect on performance. That is, $t$ tests performed across subjects at each missing age resulted in significant mean differences between obtained and expected frequencies at only 3 of the 13 age categories. More importantly, up to and including the age of eight (where over $99 \%$ of all correct responses occurred), no significant $t$ tests were found. As a result, when the average number of trials necessary to report a missing item was compared with an expected number for each sub- 
ject, the mean difference was not significantly different from zero. Because performance on any given trial was, then, essentially independent of the number of trials on which an item had remained missing, one can conclude that: (1) a best choice based on missing number age was not used, and therefore (2) a weakest-first hypothesis in which subjects were biased to select the oldest number (or number with the weakest memory representation) as their first response cannot account for the first-choice superiority found in the present experiment.

Additional evidence against a weakest-first hypothesis was also generated by directly observing the age of items selected for first and second choices. For example, the strongest evidence was uncovered by noting that when only one response was correct, the mean age of the overlooked number did not significantly differ from (and tended to be older than) the mean age of the reported number. Thus, it seems clear that missing-number success had little to do with the age or memory strength of missing numbers. In fact, because response age was also equivalent when both choices were correct (and tended to be younger for the first choice), whether the older or younger missing number was chosen first must be due to other factors.

Although these results were counter to the improved short-term memory recall of repeated items that has been found in many studies, even when the items were repeated across separate trials and subjects believed that prior lists were irrelevant to their performance on a given list (e.g., Cunningham, Healy, \& Williams, 1984; Hebb, 1961; Kintsch, 1965), studies with methods similar to those used here also did not yield a beneficial effect of repetition. The study most similar to ours was conducted by Alan Bostrom and Gordon Bower (G. H. Bower, personal communication, May 12, 1988; see also Kimble, 1967, pp. 42-43). That study included a missing-one experiment in which the same digit was kept missing until it was correctly identified. Bostrom and Bower examined whether the probability of a correct response was stationary and constant, and thus independent of the number of prior presentations of a digit string to which the subject had responded incorrectly. They found reasonably constant, stationary correct-response probabilities following 0,1 , 2 , or 3 successive errors on a digit string in which the order of digits varied but the missing digit was the same. Bostrom and Bower concluded that there was no accumulative incremental learning of the missing digits.

In a well-known attempt to understand how repetition affects associative learning, Rock (1957) similarly concluded that the formation of associations could not be explained by a process of gradual strengthening as a function of repetition. In a task that required subjects to learn a list of 12 letter-number pairs (in Experiment 1), or 12 pairs of three-letter nonsense syllables (in Experiment 2), an experimental group in which only successfully completed pairs were repeated on the next trial was compared with a control group. And, by discovering that the mean number of trials necessary to obtain one errorless trial did not differ across groups, Rock reasoned that the control subjects must not have benefited from the repetition of pairs in the initial formation of the necessary associations. Despite an often cited criticism that Rock's conclusions were confounded by the possibility that replacement of only missed (and presumably more difficult) associations provided subjects an opportunity to select easier tobe-learned items (see, e.g., Underwood, Rehula, \& Keppel, 1962), several subsequent studies have nevertheless controlled for this potential confounding and in the process have confirmed Rock's conclusions (Clark, Lanceford, \& Dallenbach, 1960; Estes, Hopkins, \& Crothers, 1960; Rock \& Heimer, 1959). For example, in a study that supported Rock's conclusion with a free-recall task, Murdock and Babick (1961) used a similar paradigm that avoided confounding due to item difficulty. Of 25 words presented for recall, one critical word was repeated on as many trials as were necessary for its recall, at which time a new critical word was introduced in its place. Two main findings demonstrated an inability of subjects to benefit from repetition. First, the probabilities of recalling critical and noncritical words were not significantly different. Second, a chi-square goodness-of-fit test that compared an obtained distribution of correct responses at each repetition with an expected geometric distribution (assuming repetition had no effect) was not significant.

Although the results from Bostrom and Bower, Rock (1957), and Murdock and Babick (1961) paralleled those of the present study, more recently Murdock and Lamon (1988) have presented evidence that made a different set of conclusions possible. In a recognition learning paradigm in which recognized words on one trial were replaced on the next trial, the repetition of unrecognized words resulted in greater recognition (relative to recognition of replacement words) than did a control condition in which no replacement took place. Although these results conflict with the research reviewed above, this difference seems explainable in terms of task differences. Specifically, it seems possible that the critical disparity existed in the prediction that was tested in each case. Whereas Murdock and Lamon upheld a prediction that repetition would benefit performance in a recognition task, in which the strongest or most familiar items would be the correct responses, the present study failed to demonstrate that the opposite prediction could be sustained in a missingnumber task, in which the weakest or least familiar items would be the correct responses.

Given the evidence counter to a weakest-first hypothesis, what, then, caused the superior first-choice performance? Because the results of the present experiment were nearly identical to those of Experiment 1 (see Table 1), one answer to this question can be found by returning to the conclusions made in the first experiment. Note that (1) the percentage of correct responses dropped dramatically from the first to the second choice, and (2) large differences between the distributions of commission errors across presentation position existed from the first to the second choice. These observations provide evidence 
for the problems associated with retrieval and support Buschke's (1963b) proposal that the higher missingnumber estimates were due to an absence of the detrimental effects of retrieval. Although it was shown in Experiment 2 that the source of these problems associated with retrieval was not output per se, neither Experiment 1 nor Experiment 2 could rule out retrieval processes prior to output as a possible source of interference. Therefore, by proposing a distinction between output interference and retrieval interference, one could explain a first-choice superiority in terms of retrieval processes.

\section{GENERAL DISCUSSION}

\section{Summary}

The missing-number task was introduced by Buschke (1963b) as a retrieval-free means of estimating the capacity of short-term memory. With only one output of the missing number, Buschke claimed that an improved estimate free from the inhibiting effects of retrieval was possible. The three experiments reported here expanded upon Buschke's work by including two missing numbers and two choices on each trial. Large performance differences between first and second choices in Experiment 1 were consistent with interference due to output and retrieval. Experiment 2 introduced the hypothesis that subjects instead had the ability to access a single best choice that was based on an inverse function of memory strength. When subjects were postcued for either a forward or a backward report order, the predicted loss of first-choice superiority was found. This finding supports either the weakest-first hypothesis or an explanation in terms of retrieval interference. The output demands of Experiment 2 may have resulted in either the item with the weakest memory representation (in accord with the weakest-first explanation) or the item that is retrieved first (in accord with an explanation in terms of retrieval interference) being reported equally often in the two response positions. The third and last experiment, then, resulted in the most surprising findings. In an attempt to find new support for a weakest-first hypothesis, we examined a prediction that subjects would select the item with the weakest memory representation as the first choice. In a paradigm that manipulated memory strength by replacing only correctly identified missing numbers, a replication of the first-choice superiority from Experiment 1 could not be explained by a bias to select the missing numbers that had been missing the longest. More importantly, because missing-number success was essentially independent of the number of trials on which an item had remained missing, evidence for a weakestfirst hypothesis was clearly absent. And because output interference was ruled out as a contributing factor in Experiment 2 , only the retrieval interference hypothesis survived all three experiments.

\section{Theoretical Analysis}

Application and tests of the missing-number technique would be facilitated if we had a better understanding of the processes involved. How is the missing-number task performed? The answer requires a broader theoretical framework or model. Currently, several competing models are broad and general enough to transcend specific experimental paradigms: the SAM model of Raaijmakers and Shiffrin (Gillund \& Shiffrin, 1984; Raaijmakers \& Shiffrin, 1981; Shiffrin \& Raaijmakers, in press), the MINERVA model of Hintzman $(1986,1988)$, the TODAM model of Murdock (1982, 1987, in press), the CHARM model of Eich $(1982,1985)$, the matrix models of Anderson (1970), Pike (1984), and Humphreys, Bain, and Pike (1989), and various connectionist models (Hinton \& Anderson, 1981; McClelland \& Rumelhart, 1981; Rumelhart \& McClelland, 1982).

It turns out that there is a natural and simple account of the missing-number task in TODAM (Murdock, in press), and we will focus on that. In any distributedmemory model, items are stored by superposition. In TODAM, for instance, items are added to a common memory vector. Furthermore, a model such as TODAM needs a working memory (WM) to carry out the associative and retrieval operations (e.g., convolution and correlation). Numbers form a highly overlearned set, and these are presumably stored in some sort of a lexicon or semantic memory.

Here is one way in which the missing-number task could be performed. The instructions specify the reference set, so the requisite numbers (here, 1-13) are summed and a representation is entered into one of the registers of WM. Then, as each list is presented, the numbers are summed and the result is placed in a second register of WM. At retrieval, the difference of these two registers is entered into a third register, and in the present case, this difference constitutes a noisy version of the sum of the two missing numbers. The noise is an inherent property of any distributed-memory model; it can be minimized (e.g., by increasing the number of elements in the item vectors that represent the stimuli), but it cannot be eliminated.

The noisy version must be deblurred (i.e., interpreted) and mapped into two separate items (the two missing numbers), and a connectionist network such as a Hopfield net (Hopfield, 1982) or the "brain-state-in-a-box" (Anderson, Silverstein, Ritz, \& Jones, 1977) would be required to deblur a linear combination of two responses. This would not be part of the distributed-memory model per se, but a separate module for the response stage (e.g., the $R$ system in TODAM).

How would such a view explain the results? In free choice (Experiments 1 and 3), by any reasonable deblurring scheme the number deblurred first would be more accurate, so first choice should have fewer errors than second choice. The disadvantage of being deblurred second can be viewed as the cause of what we have been calling retrieval interference. That is, the process used to deblur, or recover, the first missing number from the third WM register may destroy some of the information needed to deblur the remaining missing number, thereby yielding omission errors even when there is no forgetting of the presented numbers. In forced choice (Experiment 2), most of the time both numbers would have to 
be deblurred before either could be reported, but the first could be stored in WM until the process is completed. No output interference would be expected because the task demands do not exceed the capacity generally ascribed to WM (four to five registers).

The recency effect is more prominent for the first-choice data than for the second-choice data because the later are noisier (due to the higher error rate). The recency effect per se is a natural consequence of how information is stored in (working) memory. TODAM uses the storage equation from the matched-filter model (Anderson, 1973); namely,

$$
\mathbf{M}_{j}=\alpha \mathbf{M}_{j-1}+\mathbf{f}_{j},
$$

where $\alpha$ is the forgetting parameter, which produces pure recency. ( $M$ is the memory vector, here a register in WM, and $f_{j}$ would be the $j$ th number in the sequence.)

TODAM can thus account for commission errors that are found in both the missing-one and missing-two tasks. If there is some forgetting of the presented items, not all of them will be included in the second WM register. As an example, if (1) the reference set consists of the 13 numbers from 1 to 13 , (2) the set of numbers presented to the subjects in a missing-two task consists of the 11 numbers $3,11,12,1,4,5,6,13,7,9$, and 10 (i.e., the numbers 2 and 8 are missing), and (3) the number 9 is forgotten, then the second WM register would include a sum of the numbers $3,11,12,1,4,5,6,13,7$, and 10 . As a result, the third WM register would include a noisy version of the sum of the numbers 2,8 , and 9 (i.e., the two missing numbers along with one of the presented numbers, which had been forgotten). A commission error would occur if 9 , rather than 2 or 8 , were deblurred from the third WM register. As mentioned earlier, the processes in the response module (or R system) used to deblur the third WM register need to be delineated, and investigations of the missing-two task should be helpful in providing relevant evidence about these processes. For example, the findings of Experiment 3 imply that memory strength is not a factor relevant to the deblurring process. Furthermore, it may be illuminating in future research to manipulate the similarity of the items in the reference set along various dimensions, including the phonological and the visual. Both the overall frequency of commission errors and the decline in performance for the second relative to the first choice should decrease if the deblurring process is facilitated by reducing the similarity of the items.

Other than a response bias (not explained by any of the models), there is no effect of the actual number in the missing-two task, and no reason why there should be. There is no effect of repetition in Experiment 3, because the process is ahistorical; that is, on each trial a new entry is stored in WM, and no cumulative record is kept.

This explanation provides a qualitative account of the findings of the present experiments but not a quantitative account. For a quantitative account, one would have to implement this description in a particular distributed mem- ory model with a particular response module and either estimate parameters or carry out the necessary simulations. However, we should emphasize that none of this is ad hoc; all the requisite processes have been suggested (and implemented) before. All that we are doing here is to combine them in a particular way to show how a missing-number task with two missing numbers could be performed.

It is not strictly necessary to have a distributed-memory model. The MINERVA model of Hintzman (1988) does not use superposition; but it is a global matching model (Humphreys, Pike, Bain, \& Tehan, 1989), and it could presumably do the same thing because the information is summed at the time of retrieval. It would be more problematic in the CHARM model of Eich (1985), because she uses autoconvolution for item vectors; deblurring in the absence of partial cues would thus be more difficult. The SAM model of Shiffrin and Raaijmakers (in press) is a computational model, not a process model, so further elaboration would be required to apply it to this particular task. Finally, the matrix models of Anderson (1970) and Humphreys, Bain, and Pike (1989) are designed more for the storage of associative than for the storage of item information, but because the latter now includes item information as well, it too could probably be applied to the missing-number task.

According to our explanation, what we have called the "weakest" item would in fact be the strongest item when the presented set is subtracted from the reference set. This is intuitively somewhat more satisfying, because it is easier to imagine how one could identify the strongest item than it is to imagine how one could identify the weakest item.

Finally, it should be pointed out that this account shows how an augmented version of Buschke's (1963b) missingscan task could be performed without any scan at all. Serial processing of two sets of different sizes to identify the items missing from the intersection is awkward and clumsy at best. We prefer instead an account that is based on a distributed memory with a response module, and it seems to be consistent with the major results from the present experiments.

\section{Conclusions}

The potential importance of research with the missingtwo task should not be underestimated, for several reasons. First, an understanding of the processes involved in this task should provide insight into the processes involved in related tasks performed outside the laboratory; for example, when a teacher administers an examination to students in a classroom (analogous to the presented set of items), he or she determines which students are absent (the missing items) from the class (the reference set). Second, the missing-two task differs from other related laboratory tasks, such as part-list cuing and single-probe procedures, because it uniquely isolates the effects of outputting one item on the retrieval and output of another item. For example, the single-probe procedure does not 
capture the interactions among the recalled items and items still unrecalled or the interactions between unrecalled items. Third, this technique might provide interesting insights into feeling-of-knowing judgments, because information is provided about which items are not known, rather than about which items are known, to have occurred in a presented set. Finally, application of the missing-two task may be critical to the study of memory development. One of the most stable findings in the developmental literature is the improvement of memory span. But to what degree this trend actually represents developmental decreases in the effects of retrieval interference (rather than increases in storage capacity) is not apparent (see Brainerd \& Reyna, 1989, for a related suggestion). Because it allows for the examination of the effects of retrieving one item on the retrieval of a subsequent item, the missing-two task is a potentially important tool for memory research. Furthermore, because it can explain the processes that occur in the missing-number task and can pinpoint the locus of retrieval interference effects in the missing-two task, the TODAM model is a potentially important theoretical framework for memory research.

\section{REFERENCES}

ANDERSON, J. A. (1970). Two models for memory organization using interacting traces. Mathematical Biosciences, 8, 137-160.

Anderson, J. A. (1973). A theory for the recognition of items from short memorized lists. Psychological Review, 80, 417-438.

Anderson, J. A., Silverstein, J. W., Ritz, S. A., \& Jones, R. S. (1977). Distinctive features, categorical perception, and probability learning: Some applications of a neural model. Psychological Review, 84, 413-451

Brainerd, C. J., \& ReYNA, V. F. (1989). Output-interference theory of dual task deficits in memory development. Journal of Experimental Child Psychology, 47, 1-18.

Brainerd, C. J., Reyna, V. F., Howe, M. L., Kevershan, J. (1990). The last shall be first: How memory strength affects children's retrieval. Psychological Science, 1, 247-252.

Brondbent, D. E. (1958). Perception and communication. New York: Pergamon.

BUSCHKE, H. (1963a). Relative retention in immediate memory determined by the missing number method. Nature, 200, 1129-1130.

BuschKR, H. (1963b). Retention in immediate memory estimated without retrieval. Science, 140, 56-57.

BUSCHKE, H., HINRICKS, J. V. (1968). Relative vulnerability of iteminformation in short-term storage for the missing number. Journal of Verbal Learning \& Verbal Behavior, 7, 1043-1048.

BuschKe, H., \& LENON, R. (1969). Ordinal sequence in short-term retention of numbers. Journal of Verbal Learning \& Verbal Behavior, 81, 201-203.

Clark, L. L., Lanceford, T. G., * Dallenbach, K. M. (1960). Repetition and associative learning. American Joumal of Psychology, 73, 22-40.

Cunningham, T. F., Healy, A. F., * Williams, D. M. (1984). Effects of repetition on short-term retention of order information. Joumal of Experimental Psychology: Learning, Memory, \& Cognition, 10, 575-597.

Dalezman, J. J. (1976). Effects of output onder on immediate, delayed, and final recall performance. Journal of Experimental Psychology: Human Learning \& Memory, 2, 597-608.

Еich, J. М. (1982). A composite holographic associative recall model. Psychological Review, 89, 627-661.
EICH, J. M. (1985). Levels of processing, encoding specificity, elaboration, and CHARM. Psychological Review, 92, 1-38.

Erucsson, K. A. (1985). Memory skill. Canadian Jourmal of Psychology, 39, 188-231.

Estes, W. K., Hopirins, B. L., \& Crothers, E. J. (1960). All-or-none and conservative effects in the learning and retention of paired associates. Joumal of Experimental Psychology, 60, 329-339.

Gillund, G., \& Shiffrin, R. M. (1984). A retrieval model for both recognition and recall. Psychological Review, 91, 1-67.

HEBB, D. O. (1961). Distinctive features of learning in the higher animal. In J. F. Delafresnaye (Ed.), Brain mechanisms and leaming (pp. 3746). New York: Oxford University Press.

Hinton, G. E., \& Anderson, J. A. (Eds.) (1981). Parallel models of associative memory. Hillsdale, NJ: Erlbaum.

HinTZMAN, D. L. (1986). "Schema abstraction" in a multiple-trace memory model. Psychological Review, 93, 411-428.

Hintzman, D. L. (1988). Judgments of frequency and recognition memory in a multiple-trace memory model. Psychological Review, 95, 528-551.

HopFIELD, J. J. (1982). Neural networks and physical systems with emergent collective computational abilities. Proceedings of the National Academy of Sciences of the United States of America, 79, 2554-2558.

Humphreys, M. S., Bain, J. D., \& Pike, R. (1989). Different ways to cue a coherent memory system: A theory for episodic, semantic, and procedural tasks. Psychological Review, 96, 208-233.

Humphreys, M. S., PuKe, R., BanN, J. D., * Tehan, G. (1989). Global matching: A comparison of the SAM, Minerva II, Matrix, and TODAM models. Journal of Mathematical Psychology, 33, 36-67.

Kimble, D. P. (Ed.) (1967). The organization of recall. New York: New York Academy of Sciences.

KINTSCH, W. (1965). The effects of repetition on the short-term memory function. Psychonomic Science, 2, 149-150.

Klapp, S. T., Marshburn, E. A., \& Lester, P. T. (1983). Short-term memory does not involve the "working memory" of information processing: The demise of a common assumption. Journal of Experimental Psychology: General, 112, 240-264.

KLAPP, S. T., NETICK, A. (1988). Multiple resources for processing and storage in short-term memory. Human Factors, 30, 617-632.

LeWANDOWSKY, S., \& MuRdock, B. B., JR. (1989). Memory for serial order. Psychological Review, 96, 25-57.

MADSEN, M. C., Drucker, J. M. (1966). Immediate memory by missing scan and modified digit span. Psychonomic Science, 6 , 283-284.

McClelland, J. L., \& Rumelhart, D. E. (1981). An interactive activation model of context effects in letter perception: Part 1. An account of basic findings. Psychological Review, 88, 375-407.

MeWhort, D. J. K., POPHAM, D. (1991). Serial recall of tachistoscopic letter strings. In W. E. Hockley \& S. Lewandowsky (Eds.), Relating theory and data: Essays on human memory in honor of Bennet B. Murdock (pp. 425-443). Hillsdale, NJ: Erlbaum.

Miller, G. A. (1956). The magical number seven, plus or minus two: Some limits on our capacity for processing information. Psychological Review, 63, 81-97.

MURDOCK, B. B., JR. (1982). A theory for the storage and retrieval of item and associative information. Psychological Review, 89, 609-626.

MURDOCK, B. B., JR. (1987). Serial-order effects in a distributed-memory model. In D. S. Gorfein \& R. R. Hoffman (Eds.), Memory and learning: The Ebbinghaus centennial conference (pp. 277-310). Hillsdale, NJ: Erlbaum.

MurDock, B. B. (in press). Serial organization in a distributed memory model. In A. F. Healy, S. M. Kosslyn, \& R. M. Shiffrin (Eds.), From leaming theory to connectionist theory: Essays in honor of William K. Estes (Vol. 1). Hillsdale, NJ: Erlbaum.

Murdock, B. B., JR., \& BABICK, A. J. (1961). The effect of repetition on the retention of individual words. American Journal of Psychology, 74, 596-601.

MURDOCK, B., \& LAmon, M. (1988). The replacement effect: Repeat- 
ing some items while replacing others. Memory \& Cognition, 16, 91-101.

PIKE, R. (1984). Comparison of convolution and matrix distributed memory systems for associative recall and recognition. Psychological Review, 91, 281-294.

RAaijmakers, J. G. W., \& Shiffrin, R. M. (1981). Search of associative memory. Psychological Review, 88, 93-134.

Reyna, V. F., Brainerd, C. J. (1989). Output interference, generic resources, and cognitive development. Journal of Experimental Child Psychology, 47, 42-46.

Rock, I. (1957). The role of repetition in associative learning. American Journal of Psychology, 70, 186-193.

Rock, I., \& HeIMER, W. (1959). Further evidence of one-trial associative learning. American Journal of Psychology, 72, 1-16.

Rumelhart, D. E., MCClellaNd, J. L. (1982). An interactive activation model of context effects in letter perception: Part 2. The contextual enhancement effect and some tests and extensions of the model. Psychological Review, 89, 60-94.
Shiffrin, R. M., RAAumakers, J. (in press). The SAM retrieval model: A retrospective and prospective. In A. F. Healy, S. M. Kosslyn, \& R. M. Shiffrin (Eds.), From learning processes to cognitive processes: Essays in honor of William $K$. Estes (Vol. 2). Hillsdale, NJ: Erlbaum.

Thorndike, R. L., Hagen, E. P., \& Sattler, J. M. (1986). The Stanford-Binet intelligence scale (4th ed.). Chicago, IL: Riverside.

Tulving, E., Patkau, J. E. (1962). Concurrent effects of contextual constraint and word frequency on immediate recall and learning of verbal material. Canadian Joumal of Psychology, 16, 83-95.

UNDERWOOD, E. J., ReHUlA, R., \& KePPEL, G. (1962). Item selection in paired-associative learning. American Journal of Psychology, 75, 353-371.

WOODWORTh, R. S., * SCHLOSBERG, H. (1954). Experimental psychalogy. New York: Rinehart \& Winston.

(Manuscript received February 4, 1991; revision accepted for publication July 26, 1991.)

\title{
Notices
}

\section{Nominations for the Editorship of Memory \& Cognition}

Nominations are solicited for the editorship of Memory \& Cognition. The term of the present editor, Margaret Jean Intons-Peterson, expires at the end of 1993. The new editor will begin an official 4-year term January 1, 1994, and will begin to receive manuscripts early in January 1993. It is expected that the Publications Committee of the Psychonomic Society will make the appointment by July 1992.

Nominations must be submitted by April 15, 1992.

Nominations (including self-nominations) should be sent to:

\author{
Alice F. Healy \\ Department of Psychology \\ University of Colorado \\ Campus Box 345 \\ Boulder, CO 80309-0345
}

\section{Nominations for the Editorship of Perception \& Psychophysics}

Nominations are solicited for the editorship of Perception \& Psychophysics. Charles W. Eriksen, who has been the editor since 1971, will be retiring in 1993. The new editor will begin an official 4-year term January 1, 1994, and will begin to receive manuscripts early in 1993. It is expected that the Publications Committee of the Psychonomic Society will make the appointment by July 1992.

Nominations must be submitted by April 15, 1992.

Nominations (including self-nominations) should be sent to:

\author{
Anne M. Treisman \\ Russell Sage Foundation \\ 112 East 64th Street \\ New York, NY 10021
}

